\title{
Apport de la technique PCR pour une meilleure compréhension de l'épizootiologie des trypanosomoses bovines : exemple de la zone d'aménagement pastoral de Yalé au Burkina Faso
}

\section{Mots-clés}

Bovin - Glossina - Trypanosomose Trypanosoma congolense - Trypanosoma simiae - Trypanosoma vivax Amplification chaîne polymérase Sonde moléculaire - Relation hôte pathogène - Vecteur de la maladie Epidémiologie - Burkina Faso.

\author{
J.M. Reifenberg ${ }^{1}$ P. Solano ${ }^{1}$ B. Bauer ${ }^{2}$ I. Kabore ${ }^{2}$ \\ G. Cuny ${ }^{3}$ G. Duvallet ${ }^{1}$ D. Cuisance ${ }^{1}$
}

\section{INTRO DUCTION}

De nos jours, le diagnostic des trypanosomoses animales africaines (TAA), parasitoses responsables de pertes considérables dans la production animale en Afrique sub-saharienne, repose sur trois groupes de techniques : parasitologiques, séro-immunolo-

1. CIRAD-EMVT, Campus international de Baillarguet, BP 5035, 34032 Montpellier Cedex 1, France

2. CIRDES, 01 BP 454 Bobo-Dioulasso 01, Burkina Faso

3. ORSTOM-LEMV, BP 5045, 34032 Montpellier Cedex 1, France giques et moléculaires. Le principe des méthodes parasitologiques est basé sur l'observation directe, au microscope, des trypanosomes chez leurs différents hôtes. La fiabilité de ces techniques provient de la mise en évidence des infections actives, leur conférant les avantages d'un diagnostic de certitude. Leur inconvénient majeur est le manque de sensibilité, car elles ne permettent pas de déceler chez l'hôte vertébré, dans les meilleures conditions, moins de 100 trypanosomes/ml de sang pour la technique de double-centrifugation (48).

Les techniques séro-immunologiques les plus utilisées reposent sur la mise en évidence, par la technique ELISA, d'anticorps sécrétés par l'hôte en réponse à l'infection, ou d'antigènes circulants 
consécutifs à la lyse des agents pathogènes par les défenses immunitaires de l'hôte. Le test ELISA de détection des anticorps chez l'hôte vertébré se montre efficace pour l'analyse de nombreux échantillons de sérums au cours d'études épidémiologiques de grande ampleur (4). Un test ELISA de détection des antigènes permettant, à la différence du précédent, de révéler les infections précoces et actives, est en évaluation depuis quelques années $(3,9)$ mais pose des problèmes de sensibilité et de spécificité $(10,11)$.

En complément des nombreuses recherches sur l'amélioration des techniques parasitologiques et séro-immunologiques pour le diagnostic des trypanosomoses animales, les techniques moléculaires offrent de très intéressantes perspectives et sont de plus en plus fréquemment utilisées pour la détection et l'identification des parasites chez les différents hôtes. Des séquences spécifiques ont été localisées dans l'ADN génomique des parasites pour différencier les principaux trypanosomes pathogènes et différentes techniques ont été développées pour les révéler (28,29). L'application de sondes spécifiques par la technique d'hybridation moléculaire chez les glossines $(14,22,27,30)$ et les bovins (37) a été peu à peu remplacée par la technique PCR (polymerase chain reaction) (44), qui consiste à mettre en évidence les mêmes régions dans le génome des parasites, non pas par hybridation moléculaire, mais par amplification génique in vitro. Par son principe, cette technique offre des possibilités inégalables en terme de spécificité et de sensibilité. Elle est néanmoins encore délicate à maîtriser en tant qu'outil de diagnostic de routine. Etant donné que la Taq Polymerase est très sensible à de nombreux facteurs, notamment les éléments sanguins et leurs dérivés, une purification correcte des échantillons est primordiale afin de limiter les inhibitions de l'amplification. Quelques techniques simples de traitement des échantillons, alternatives à la procédure fastidieuse de l'extraction d'ADN, ont montré d'excellents résultats $(30,38,46,50)$.

Transférées au CIRDES (Centre international de recherche-développement sur l'élevage en zone subhumide) en 1992, ces biotechnologies, après une période d'adaptation, ont été utilisées à plusieurs reprises en conditions naturelles (42, 46, 47). L'étude présentée ici se situe dans la lignée des travaux entrepris antérieurement sur le terrain au Burkina Faso. Elle a été réalisée dans le cadre d'un programme de lutte contre les trypanosomoses, engagé dans la zone d'aménagement pastoral de Yalé, située dans la province de la Sissili, au sud du pays. Son intérêt, grâce aux biotechnologies, est d'apporter aux connaissances acquises par les techniques classiques des éléments supplémentaires de compréhension sur la nature et la circulation des trypanosomes pathogènes dans ce foyer actif de la maladie. Les résultats de ce travail ont été comparés à d'autres obtenus en conditions naturelles et expérimentales et les implications d'ordre fondamental et appliqué sont alors discutées.

\section{MATERIEL ET METHODES}

\section{Contexte de l'étude}

Les échantillons ont été récoltés au cours des missions des équipes de l'Unité de lutte contre les maladies parasitaires et leurs vecteurs du CIRDES (2), dans le cadre d'un programme de lutte contre les trypanosomoses, responsables à l'époque d'une régression alarmante du cheptel bovin dans la zone d'aménagement pastoral de Yalé, située dans la province de la Sissili, au sud du Burkina Faso.

\section{Situation géographique}

La province de la Sissili est située dans la zone climatique sudsoudanienne qui regroupe les régions localisées à l'ouest et à l'est du Mouhoun. La zone sud-soudanienne est constituée de forêts claires et de savanes traversées de galeries forestières, véritables formations hygrophiles plus ou moins denses, plus ou moins larges, composées d'essences diverses. La végétation est fortement influencée par les feux de brousse.

Les sites de prospection se situent à proximité du ranch de gibier de Nazinga qui est localisé aux environ de $11^{\circ} 9^{\prime}$ de latitude $\mathrm{N}$ et $1^{\circ} 30^{\prime}$ de longitude O. Il abrite l'un des derniers noyaux de faune sauvage du pays : Sylvicapra grimmia L. (céphalophe de Grimm), Ourebia ourebi Zimmermann (ourébi), Hippotragus equinus Desmarest (hippotrague), Loxodonta africana Brummenbach (éléphant), Phacocherus aethiopicus Pallas (phacochère), Tragelaphus scriptus Pallas (guib harnaché), Kobus ellipsiprymnus Ogilby (cob defassa), des singes, quelques carnivores et de nombreux reptiles (crocodiles, varans, serpents).

Trois espèces de glossines occupent cette zone. G. tachinoides, prédominante, G. palpalis gambiensis et G. morsitans submorsitans. L'espèce dominante en effectif, capturée le long des lignes de drainage dans les zones de contact glossines-troupeaux de bovins, fut $G$. tachinoides.

\section{Récolte des échantillons de glossines}

Les glossines ont été capturées grâce à deux types de piège : le piège biconique Challier-Laveissière, décrit en 1976 (24), et le piège monoconique «Vavoua » (25). Les insectes ont été disséqués selon la technique classique (17). Afin d'éviter les contaminations, les instruments de dissection ont été soigneusement nettoyés entre les mouches et entre les organes de chaque mouche, à raison d'un bain dans de l'eau de Javel et d'un bain dans de l'eau distillée. Après observation microscopique du proboscis, des glandes salivaires et de l'intestin moyen pour la recherche des trypanosomes, les organes des individus positifs ont été repris et suspendus dans $50 \mu \mathrm{l}$ d'eau stérile. Quatre-vingt-quatre G. tachinoides ont ainsi été retenues, parmi lesquelles un seul individu présentait une infection mature (présence de trypanosomes à la fois dans l'intestin moyen et le proboscis), les autres mouches arborant toutes des infections de l'intestin moyen uniquement.

\section{Récolte des échantillons de bovins}

Les échantillons de sang de bovins infectés ont été prélevés au cours du suivi sanitaire des 200 à 300 bovins répartis dans les différentes unités de la zone d'aménagement pastoral de Yalé. La centrifugation d'un microtube capillaire rempli du sang de chaque animal a permis la lecture de l'hématocrite, indice significatif de la trypanosomose chez les bovins. Ensuite, le buffy coat (49) a été étalé entre lame et lamelle et examiné à l'état frais au microscope en contraste de phase pour la recherche des trypanosomes. Un second tube capillaire a été préparé à partir du sang des animaux parasitologiquement positifs, et le buffy coat récupéré dans un tube Eppendorf contenant $50 \mu \mathrm{l}$ d'eau stérile pour l'analyse par PCR.

Quatre-vingt-douze échantillons ont ainsi été retenus, dont 44 étaient parasitologiquement positifs. Les 48 cas restants ne présentaient aucun trypanosome à l'observation microscopique du buffy coat.

\section{Préparation des échantillons}

Les suspensions contenant les intestins moyens de glossines et les buffy coat de bovins ont été purifiés selon une technique basée sur l'utilisation d'un kit commercial de purification des échantillons biologiques (Ready Amp ${ }^{\mathrm{TM}}$ genomic purification kit, Madison, WI, USA) (38). 
Les suspensions contenant les proboscis dans $50 \mu \mathrm{l}$ d'eau stérile ont été simplement agitées énergiquement, sans aucun traitement préalable, dans le but de faciliter la libération des trypanosomes dans le liquide environnant.

Deux à $3 \mu \mathrm{l}$ du surnageant de chaque échantillon après traitement ont été ensuite ajoutés au tampon réactionnel pour l'identification des trypanosomes par PCR.

\section{PCR}

Le protocole de la technique PCR émane de celui qui fut initialement mis au point par Masiga et coll., 1992 (33), dans un volume réactionnel de $25 \mu \mathrm{l}$ composé des réactifs suivants (concentration finale) : $10 \mathrm{mM}$ Tris- $\mathrm{HCl} \mathrm{pH} 8,3,50 \mathrm{mM} \mathrm{KCl}, 1,5 \mathrm{mM} \mathrm{MgCl}_{2}$, $200 \mu \mathrm{M}$ de dNTPs, $100 \mathrm{ng} / \mu \mathrm{l}$ d'amorces, 1,25 unités de Taq Polymerase et 2 à $3 \mu \mathrm{l}$ d'ADN. Les amorces oligonucléotidiques utilisées au cours de cette étude figurent dans le tableau I.

Une étape de dénaturation initiale à $92^{\circ} \mathrm{C}$ pendant 5 min a été suivie de 30 cycles, avec pour chacun trois étapes successives : une étape de dénaturation à $94^{\circ} \mathrm{C}$ pendant $1 \mathrm{~min}$, une étape d'hybridation des amorces à $56^{\circ} \mathrm{C}$ pendant 1 min et une étape d'élongation à $72^{\circ} \mathrm{C}$ pendant $1 \mathrm{~min}$. Le programme s'est achevé par une élongation terminale à $72^{\circ} \mathrm{C}$ durant $5 \mathrm{~min}$. Un volume de $10 \mu \mathrm{l}$ de chaque échantillon a été ensuite révélé par électrophorèse dans un gel à 2 p. 100 d'agarose teinté au bromure d'éthidium, et observé sous lumière UV. La fiabilité de chaque réaction d'amplification a été contrôlée par un témoin positif (adjonction au volume réactionnel de $50 \mathrm{pg}$ d'ADN purifié de la souche clonale de référence) et un témoin négatif (tampon réactionnel contenant tous les constituants nécessaires à l'amplification, mais sans $\mathrm{ADN}$ cible).
Les échantillons de glossines ont été analysés par PCR en utilisant les amorces spécifiques de T. congolense types « savane », « forêt », « Kilifi » et « Tsavo », T. simiae, T. brucei sensu lato et $T$. vivax.

Les échantillons de bovins ont été testés avec les mêmes amorces, à l'exception de celles reconnaissant $T$. simiae. Une prospection plus restreinte, dirigée sur les types « savane » et «forêt » de $T$. congolense, $T$. vivax et $T$. brucei s. 1., a été menée sur les échantillons parasitologiquement négatifs.

\section{RESU LTATS}

\section{Analyse des échantillons de glossines}

Parmi les 84 intestins positifs testés (positifs par les techniques parasitologiques), 50 (59,5 p. 100) ont pu être identifiés par PCR. Les taxons mis en évidence sont $T$. congolense types « savane » et « forêt »,T. simiae et $T$. vivax. Des infections mixtes associant $T$. congolense types « savane » et «forêt », T. congolense type « savane » et $T$. simiae, $T$. simiae et $T$. vivax, ont également été révélées (figure 1).

Une amplification spécifique de $T$. simiae et de $T$. vivax a été obtenue à partir des métacycliques présents dans le seul proboscis trouvé infecté par l'examen microscopique. La présence de $T$. simiae a aussi été révélée dans le contenu intestinal de la même glossine.

Parmi les 34 infections intestinales qui n'ont pu être caractérisées, certaines ont entraîné des réactions d'amplification atypique avec les amorces spécifiques de T. brucei s. l. (séquence « ingi »). Ce

\section{Tableau I}

Amorces utilisées et taille des produits d'amplification attendus

\begin{tabular}{|c|c|c|c|}
\hline Espèce/taxon & Séquence oligonucléotidique & $\begin{array}{c}\text { Taille de } \\
\text { l'amplification } \\
\text { (pb) }\end{array}$ & Référence \\
\hline $\begin{array}{l}\text { T. congolense } \\
\text { « savane » }\end{array}$ & $\begin{array}{l}\text { ILO } 344: \text { 5' CGAGCGAGAACG GGCAC 3' } \\
\text { ILO } 345: 5 \text { 5'GGGACAAACAAATCCCGC 3' }\end{array}$ & 320 & $\begin{array}{l}\text { Majiwa et O tieno, } 1990 \\
\text { (Mol. Biochem. Parasitol.) }\end{array}$ \\
\hline $\begin{array}{l}\text { T. congolense } \\
\text { « Kilifi » }\end{array}$ & $\begin{array}{l}\text { TCK1 : 5' GTGCCCAAATTTGAAGTGAT 3' } \\
\text { TCK2 : 5'ACTCAAAATCGTGCACCTCG 3' }\end{array}$ & 294 & $\begin{array}{l}\text { Masiga et coll., } 1992 \\
\text { (Int. J. Parasitol.) }\end{array}$ \\
\hline $\begin{array}{l}\text { T. congolense } \\
\text { «forêt » }\end{array}$ & $\begin{array}{l}\text { TCF1 : 5' GGACACGCCAGAAGGTACTT 3' } \\
\text { TCF2 : 5' GTTCTCGCACCAAATCCAAC 3' }\end{array}$ & 350 & $\begin{array}{l}\text { Masiga et coll., } 1992 \\
\text { (Int. J. Parasitol.) }\end{array}$ \\
\hline $\begin{array}{l}\text { T. congolense } \\
\text { «Tsavo» }\end{array}$ & $\begin{array}{l}\text { ILO } 892: \text { : 5' CGAGCATGCAGGATGGCCG 3' } \\
\text { ILO } 893: 5^{\prime} \text { GTCCTGCCACCGAGTATGC 3' }\end{array}$ & 400 & $\begin{array}{l}\text { Majiwa et coll., } 1993 \\
\text { (Parasitology) }\end{array}$ \\
\hline T. simiae & $\begin{array}{l}\text { TSM } 1: 5^{\prime} \text { CGGTCAAAAACGCATT 3' } \\
\text { TSM } 2: 5^{\prime} \text { AGTCG CCCGGAGTCGAT 3' }\end{array}$ & 437 & $\begin{array}{l}\text { Majiwa et O tieno, } 1990 \\
\text { (Mol. Biochem. Parasitol.) }\end{array}$ \\
\hline $\begin{array}{l}\text { T. brucei } \\
\text { « ingi » }\end{array}$ & $\begin{array}{l}\text { ILO } 342: 5^{\prime} \text { GATCCG CAG CCG GGCCTG 3' } \\
\text { ILO } 242: 5^{\prime} \text { CCGCGGTGG CTCCTTCCC 3' }\end{array}$ & 1530 & $\begin{array}{l}\text { Kimmel et coll., } 1987 \\
\text { (Mol. Cell. Biol) }\end{array}$ \\
\hline $\begin{array}{l}\text { T. vivax } \\
\text { Afrique de l'O uest }\end{array}$ & $\begin{array}{l}\text { TVW } 1: 5^{\prime} \text { CTGAGTGCTCCATGTGCCAC 3' } \\
\text { TVW } 2: 5^{\prime} \text { CCACCAGAACACCAACCTGA 3' }\end{array}$ & 150 & $\begin{array}{l}\text { Masiga et coll., } 1992 \\
\text { (Int. J. Parasitol.) }\end{array}$ \\
\hline
\end{tabular}



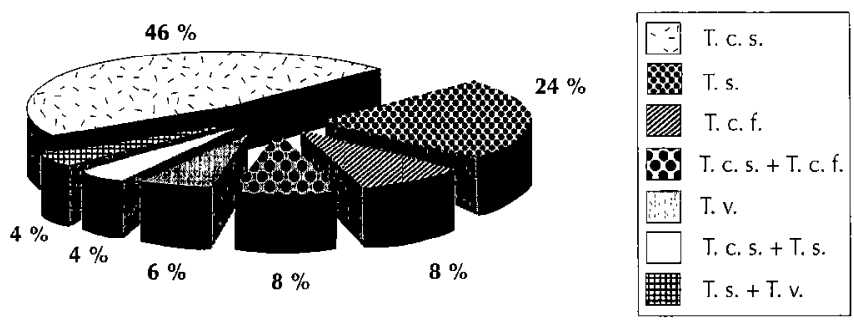

Figure 1 : trypanosomes identifiés par PCR dans l'intestin moyen de G. tachinoides $(n=84)$, zone d'aménagement pastoral de Yalé, Burkina Faso.

T.c.s. : T. congolense typc "savane "; T.c.f. : T. congolensc type "forêt »;T.s. : T. simiae; T.v. :T. vivax.

signal a ćté constaté sur plusicurs échantillons intestinaux testés avec ces amorces, ce qui pourrait témoigner de la présence d'un trypanosome « inconnu » commun à plusieurs glossines.

Parmi les 83 proboscis trouvés indemnes de trypanosomes au microscope, 8 ont montré des signaux d'amplification par PCR, dont 2 à $T$. congolense type « savane », 2 à $T$. simiae et 4 à $T$. vivax.

\section{Analyse des échantillons de bovins}

Parmi les 44 prélèvements de sang infecté, l'identification parasitologique des trypanosomes a révélé 26 cas d'infection à $T$. congolense, 17 à $T$. vivax, et 1 infection mixte à $T$. congolense et T. vivax. Aucune infection à $T$. brucei n'a été observée.

Sur les 26 infections du type Nannomonas, 22 (84,6 p. 100) ont été identifiées par PCR comme appartenant à $T$. congolense type " savane »; sur les 17 infections du type Duttonella, 7 (41,1 p. 100) seulement ont pu être confirmées par PCR. Quatorze échantillons (4 infectés par T. congolense et 10 par $T$. vivax) n'ont donc donné aucun signal d'amplification avec les amorces utilisées. La présence de T. vivax n'a pas été confirmée par PCR dans l'échantillon où une infection mixte à $T$. congolense et $T$. vivax avait été préalablement observée au microscope.

Sur les 48 cas parasitologiquement négatifs, 7 ont donné des signaux d'amplification avec les amorces spécifiques de $T$. congolense type "savane ». Sur ces 7 animaux, 5 présentaient un hématocrite inférieur à 25 , valeur limite correspondant à un état encore physiologiquement satisfaisant, les deux bovins restants ayant respectivement un hématocrite de 26 et 27.

Les techniques parasitologiques n'ont pas révélé $T$. brucei, ce que confirme la technique PCR : aucun signal n'est apparu avec les amorces spécifiques du sous-genre Trypanozoon (tableau II).

\section{DISCUSSION}

Le faible taux d'infections matures chez $G$. tachinoides corrobore des observations déjà décrites sur le terrain (41), confirmées récemment au laboratoire $(39,40,41)$. La majorité des glossines infectées avaient un âge supérieur à 30 jours. Sachant que la durée moyenne du développement cyclique de $T$. congolense est de 14 jours environ et celle de $T$. vivax de 10 jours environ, la majorité des infections acquises au moment du premier repas sanguin (la réceptivité d'une glossine étant fortement liée à son état ténéral) devrait théoriquement avoir atteint le stade infectant. Il est donc probable que le fort taux d'infections immatures soit plus associé à des incompatibilités physiologiques entre les trypanosomes et les glossines étudiées.

La majorité des infections intestinales identifiées par PCR sont causées par des trypanosomes appartenant au sous-genre Nannomonas (T. congolense types « savane » et « forêt », T. simiae) qui arrivent difficilement à maturité, comme en témoigne le très faible pourcentage de proboscis infectés. Parmi les glossines analysées par PCR, la seule qui présentait des trypanosomes dans la trompe s'est avérée infectée à la fois par $T$. vivax et par $T$. simiae. La notion selon laquelle les glossines du groupe palpalis sont réputées être des vecteurs peu efficaces des trypanosomes du sousgenre Nannomonas mérite d'être nuancée dans certaines situations. Force est de constater qu'un nombre non négligeable d'infections à $T$. simiae ont été détectées, corroborant d'autres résultats récents révélant aussi sa présence, en terme d'infections matures, dans certaines populations de G. p. gambiensis et de G. tachinoides peuplant les bordures du Mouhoun, au Burkina Faso (47).

La proximité du ranch de gibier de Nazinga implique des rencontres permanentes ou saisonnières entre les populations de glossines et la faune sauvage qui sillonne les alentours de la zone agro-pastorale. Les suidés domestiques et sauvages forment un réservoir naturel de trypanosomes, dont $T$. simiae qui est particulièrement inféodé à ces mammifères (7). La présence de $T$. simiae dans les organes de $G$. tachinoides résulte probablement de contacts fréquents avec cet hôte dans les gîtes favorables. Cette espèce oriente facilement ses attaques vers les porcs domestiques des villages autour desquels elle abonde $(1,24)$ et vers les suidés sauvages dans les zones moins anthropisées (16). Il est bien connu que l'opportunisme alimentaire de $G$. tachinoides lui confère des capacités d'adaptation aux périodes de restriction temporaire en hôtes nourriciers habituels, d'où les variations saisonnières des préférences trophiques fréquemment constatécs $(5,23,24)$. Les reptiles, tout particulièrement les varans et les crocodiles, connus comme hôtes nourriciers communs de $G$. tachinoides (24), hébergent des trypanosomes inoffensifs pour les mammifères ( $T$. varani, T. grayi-like) (35), dont le cycle évolutif est postérograde, avec un développement limité à l'intestin des vecteurs. L'analyse des repas de sang d'un échantillon de $G$. tachinoides originaire de la même région a montré que 55 p. 100 de ces repas provenaient de reptiles (Bauer B., comm. pers.). Une fraction des infections intestinales chez G. tachinoides relève probablement de ces trypanosomes, présomption confortée par le nombre élevé d'infections intestinales qui n'ont pu être caractérisées avec les marqueurs moléculaires utilisés.

Il est tentant d'avancer l'hypothèse que $T$. congolense type "forêt " dispose d'un spectre d'hôtes vertébrés différent de celui du taxon " savane ". Alors que sa présence a été détectée à plusieurs reprises chez $G$. tachinoides, il est totalement absent des prélèvements de bovins, ce qui pourrait conforter l'hypothèse d'un pouvoir pathogène plus faible que celui de $T$. congolense type " savane " pour les bovidés. La circulation de ce taxon dans cette région pourrait alors être liée à la faune sauvage, comme le guib harnaché, connu pour être très souvent sollicité $(24,34)$. L'hypothèse selon laquelle les populations locales de phacochères sont également un réservoir naturel de ce parasite mériterait d'être étudiée, d'autant que la majorité des souches connues isolées antérieurement proviennent de porcs domestiques $(14,33,45)$. 
Tableau II

Bilan de l'analyse par PCR des infections observées en microscopie dans les organes de G. tachinoides (zone d'aménagement pastoral de Yalé)

\section{Intestins positifs par examen parasitologique}

Intestins positifs par PCR
Identification

Nombre de chaque parasite

$23(46 \%)$

$12(24 \%)$

T. S.

$4(8 \%)$

T. c. f.

$4(8 \%)$

T. V.

$3(6 \%)$

T. C. S. +T. S.

T. S. + T. v.

\section{Intestins négatifs}

par PCR

$34(40,5 \%)$

Identification

Nombre de chaque parasite

\section{examen parasitologique}

Proboscis positifs par PCR
T. v. + T. s.
L'identification de T. vivax dans le contenu intestinal de plusieurs glossines témoigne vraisemblablement de l'absorption récente d'un repas sanguin prélevé sur un animal infecté, compte tenu du fait que le cycle de $T$. vivax est limité à la région cibariale et au proboscis des glossines (36). Cette constatation pose le problème de la positivité du test, suite à l'amplification par PCR d'ADN de trypanosomes récemment ingérés ou de résidus de trypanosomes subsistant dans l'intestin des glossines. La fréquence de tels résultats peut entraîner des interprétations épidémiologiques erronées, notamment dans les foyers actifs de trypanosomose où les contacts glossines-hôtes vertébrés sont fréquents. Il serait d'un grand intérêt de quantifier expérimentalement chez la glossine les infections intestinales avortées et détectables par PCR, afin d'apporter plus d'objectivité à l'interprétation de certains résultats de terrain.

Plusieurs infections mixtes avec les deux taxons de $T$. congolense ont été détectées chez $G$. tachinoides. Cette espèce de glossine affectionne une gamme variée de biotopes alors que la majorité des glossines du groupe palpalis sont plutôt riveraines. La présence concomitante de ces deux taxons a déjà été décrite chez $G$. longipalpis en Côte d'Ivoire (46) et chez G. pallidipes (50) au Zimbabwe. Ces glossines, bien qu'appartenant au groupe de G. morsitans qui réunit en majorité des espèces savanicoles, se distinguent par une plus grande ubiquité des gîtes occupés. La niche écologique de ces espèces, ni strictement savanicoles, ni strictement ripicoles, favorise la diversification des sources de nourriture, et en conséquence l'hétérogénéité parasitaire observée chez ces populations de vecteurs. Certains auteurs ont suspecté l'existence de relations d'affinités associant les glossines du groupe morsitans et le type «savane » de $T$. congolense, et le groupe palpalis et le type «forêt » de T. congolense (27). Il semblerait en fait que ce modèle hypothétique soit plus complexe : le taxon « forêt » serait transmis plus spécifiquement par les espèces de glossines vivant dans les biotopes humides et le type « savane » aurait un spectre vectoriel moins restrictif. Dans les zones infestées uniquement de glossines riveraines ou forestières $(27,45$, I. Morlais, comm. pers.), seul le type « forêt » circulerait. Le type « savane » prédominerait dans les aires de savane mais il serait également transmis par de nombreuses autres espèces de glossines aux niches écologiques plus diversifiées. Les espèces de glossines ni strictement savanicoles (c'est le cas de $G$. longipalpis et de G. pallidipes dans le groupe morsitans ), ni strictement forestières (c'est le cas de G. tachinoides dans le groupe palpalis) pourraient transporter simultanément ces deux taxons, alors qu'ils auraient tendance à s'exclure mutuellement chez les espèces de glossines aux caractéristiques écologiques plus tranchées (figure 2).

Ces observations sont à rapprocher des différences de virulence chez les mammifères domestiques abordées précédemment, le taxon «forêt » apparemment prédominant chez le porc domestique élevé surtout dans les régions forestières, le taxon « savane » paraissant plus pathogène pour les bovidés, surtout élevés dans les 


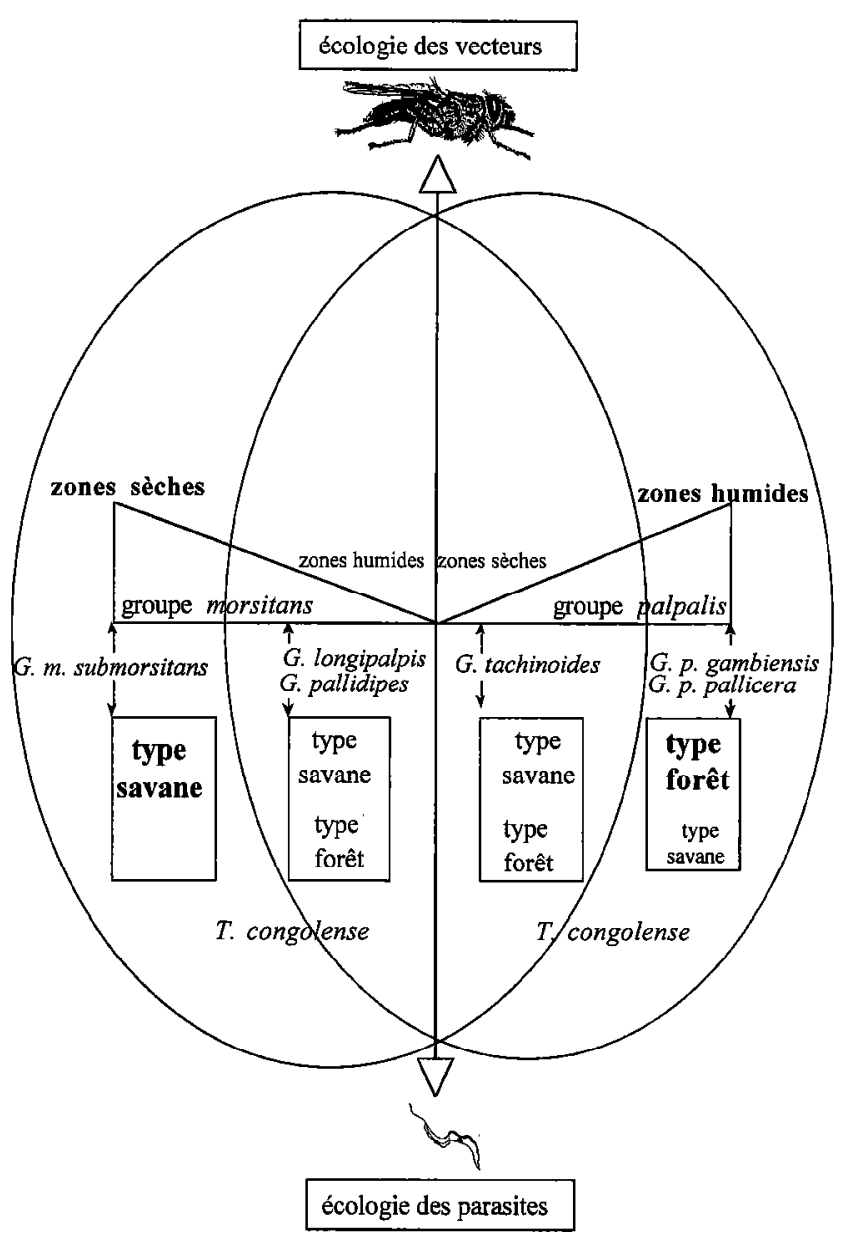

Dessin de la glossine : F. D'Amico, 1993

Figure 2 : relations d'affinité entre les taxons "savane " et " forêt " de $\mathrm{T}$. congolense et les espèces de glossines en fonction de leur habitat : schéma représentant les tendances qui se dégagent d'après les observations sur le terrain. Les glossines de zones sèches transmettraient préférentiellement $\mathrm{T}$. congolense "savane " (à gauche), les glossines de zones humides transmettraient préférentiellement T. congolense "forêt " (à droite), les glossines ni strictement savanicoles, ni strictement forestières pourraient transporter les deux taxons (au centre).

zones plus arides. Les marqueurs moléculaires utilisés à des fins diagnostiques pourraient donc avoir aussi une valeur de marqueurs de pathogénicité.

Les amplifications non spécifiques obtenues à partir des trypanosomes observés microscopiquement dans plusieurs échantillons, avec des amorces reconnaissant théoriquement le sous-genre Trypanozoon ne présagent en rien de l'identité taxonomique de ces trypanosomes intestinaux. La fréquence de ces amplifications pourrait témoigner de la présence du même trypanosome « inconnu » chez plusieurs individus.

L'hypothèse de la circulation de trypanosomes pathogènes non identifiables avec les marqueurs moléculaires connus est plausible. Outre les souches de T. vivax qui n'ont pas réagi avec les amorces usuelles, quatre isolats de sang de bovins infectés par T. congolense ne correspondent à aucun des taxons connus. Les amorces spécifiques de T. godfreyi (31) n'ont pas été testées sur les échan- tillons de cette étude. Compte tenu de la proximité taxonomique de ce taxon avec $T$. simiae et d'une répartition apparemment étendue sur tout le continent africain, l'éventualité de sa présence dans les sites d'étude des auteurs est aussi envisageable. Les éventuels problèmes techniques, comme des inhibitions résiduelles de l'amplification susceptibles d'être également responsables des problèmes rencontrés dans l'identification de certaines infections, ne peuvent, bien sûr, être exclus.

Une proportion non négligeable $(9,6$ p. 100) de proboscis négatifs à l'examen parasitologique se sont révélés positifs par PCR. Il parait peu probable que des trypanosomes soient passés inaperçus au contrôle microscopique du proboscis, à moins qu'ils n'aient été localisés dans la région cibariale (18) et aient diffusé dans des parties dissimulées de la trompe au moment de la dissection. La moitié de ces amplifications sont spécifiques de $T$. vivax. L'initiation du cycle de ce trypanosome au niveau du cibarium et de l'œsophage de la glossine (36) pourrait expliquer le plus grand nombre de cas de détection par PCR de $T$. vivax dans des proboscis de glossines parasitologiquement négatives (32).

L'analyse des prélèvements sur des animaux montre que la majorité des infections sont dues à $T$. vivax et $T$. congolense type « savane ». De nombreuses souches de $T$. vivax n'ont pas pu être identifiées par PCR. Une sensibilité hypothétiquement moyenne du marqueur moléculaire utilisé (14) et les inhibitions éventuelles ont paru insuffisantes aux auteurs pour justifier que 41,1 p. 100 des infections du type Duttonella, parmi lesquelles figuraient des infections massives, n'aient donné aucun signal positif par PCR avec les amorces spécifiques testées. Certaines souches de $T$. vivax circulant dans cette région pourraient ne pas être reconnues par les marqueurs moléculaires utilisés. Des observations similaires en Côte d'Ivoire chez G. longipalpis ont montré que seulement 35 p. 100 des infections présumées Duttonella ont été identifiées (46).

Des signaux d'amplification avec les amorces spécifiques de $T$. congolense type « savane » ont été obtenus à partir d'échantillons provenant de sept bovins aparasitémiques, mais pour la plupart suspectés d'être infectés du fait d'un hématocrite faible et d'un état général faible. Ces animaux exprimaient vraisemblablement une infection naissante ou chronique en phase aparasitémique, indécelable à l'examen microscopique, soulignant l'apport de la technique PCR pour révéler les pauci-infections (19).

\section{- CONCLUSION}

De nombreuses autres études s'avèrent nécessaires pour perfectionner la technique PCR dans le but d'obtenir un outil de diagnostic utilisable de manière plus routinière. La préparation d'échantillons à analyser, étape clé dans le protocole car elle conditionne en grande partie la reproductibilité de la technique, mérite d'être encore améliorée pour approcher la sensibilité obtenue avec de l'ADN purifié. L'étude présentée ici a montré qu'une recherche plus fondamentale et plus exhaustive paraît aussi nécessaire pour mettre au point des marqueurs diagnostiques plus « universels » permettant d'identifier la majorité des souches pathogènes, mais également des trypanosomes non pathogènes responsables des difficultés d'analyse des infections chez le vecteur (15).

En dépit de ces lacunes, cette technologic apportc, grâcc à la multiplication des applications sur le terrain, de nombreux éléments nouveaux contribuant à la compréhension de l'épizontiologie. D'un point de vue plus fondamental, elle a permis de mieux défi- 
nir les relations entre les parasites et leurs vecteurs, en précisant la capacité vectorielle de certaines espèces de glossines, en mettant en évidence les nombreuses infections mixtes chez les différents hôtes, en révélant des couples préférentiels parasites-vecteurs. De telles contributions sont d'une utilité réelle pour permettre de mieux cibler la lutte antivectorielle et de mieux évaluer les conséquences de la lutte contre cette endémie qui demeure une des premières préoccupations de l'élevage en Afrique sub-saharienne.

\section{Remerciements}

Les auteurs tiennent à remercier tout particulièrement Dr S. M. Touré, directeur général du CIRDES, pour avoir autorisé cette étude conjointement avec les équipes de l'Unité de lutte contre les maladies parasitaires et leurs vecteurs, ainsi que Dr J.L. Frézil, responsable du Laboratoire d'épidémiologie des maladies à vecteurs du Département santé de l'ORSTOM, pour son accueil au sein de son laboratoire.

\section{BIBLIO GRAPHIE}

1. BALDRY D.A.T., 1964. Observations on a close association between Glossina tachinoides and domestic pigs near N sukka, Eastern Nigeria II Ecology and trypanosome infection rates in G. tachinoides. Ann. trop. Med. Parasitol., 58: 32-44.

2. BAUER B., 1993. Enquêtes entomologiques et épidémiologiques dans la zone pastorale de Yalé (Sissili). Bobo-Dioulasso, Burkina Faso, CIRDES, 25 p. (Série de 6 rapports de missions)

3. BENGALY Z., GAUTHIER J., ARGIRO L., DELAFO SSE A. DUVALLET G., 1994. Evaluation of antigen detection ELISA for the diagnosis of trypanosomosis in experimentally and naturally infected livestock. In: First research coordination meeting on "use of immunoassay methods for improved diagnosis of trypanosomiasis and monitoring tsetse and trypanosomiasis control programmes", ILRAD, Nairobi, Kenya, February 7-11, 1994.

4. BOCQUENTIN R., DUVALLET G., 1990. Amélioration de la reproductibilité du test ELISA adapté à la détection d'anticorps antiTrypanosoma congolense chez les bovins. Revue Elev. Méd. vét. Pays trop., 43 : 179-186.

5. BO REHAM P.F.L., GILL G.S., 1973. Serological identification of reptile feeds of Glossina. Acta trop., 30: 356-365.

6. CHALLIER A., LAVEISSIERE C., 1973. U n nouveau piège pour la capture des glossines (Glossina : Diptera, Muscidae), description et essais sur le terrain. Cah. ORSTOM, Sér. Ent. méd. Parasitol., 11 : 251-262.

7. CLAXTON J.R., FAYE J.A., RAW LIN GS P., 1992. Trypanosome infections in warthogs (Phacochoerus aethiopicus) in The Gambia. Vet. Parasitol., 41: 179-187.

8. D'AMICO F., 1993. Rôle de Glossina fuscipes fuscipes Newstead 1910 dans la transmission des trypanosomoses bovines en Afrique Centrale. Cas de la zone d'élevage d'Ouro-Djafoun. Thèse doct., U niversité Montpellier II, Montpellier, France, $160 \mathrm{p}$.

9. DELAFO SSE A., BENGALY Z., DUVALLET G., 1996. U tilisation du test ELISA de détection des antigènes circulants de trypanosomes dans la cadre d'un suivi épidémiologique dans la zone de Sidéradougou, Burkina Faso. Revue Elev. M éd. vét. Pays trop., 49 : 32-37.

10. DESQ UESNES M., 1996. Evaluation of three antigen detection tests (monoclonal trapping ELISA) for African trypanosomes, with an isolate of Trypanosoma vivax from French Guyana. Ann. N. Y. Acad. Sci., 791: 172-184.
11. DESQ UESNES M., DE LA ROCQUE S., 1995. Comparaison de la sensibilité du test de Woo et d'un test de détection des antigènes de Trypanosoma vivax chez deux moutons expérimentalement infectés avec une souche guyanaise du parasite. Revue Elev. Méd. vét. Pays trop., 48 : 247-253.

12. GIDUDU A.M., CUISAN CE D., REIFEN BERG J.M., FREZIL J.L., 1995. Contribution à l'étude de l'émission de Trypanosoma congolense par Glossina morsitans morsitans (Diptera : Glossinidae) au laboratoire. Revue Elev. Méd. vét. Pays trop., 48 : 264-270.

13. GIDUDU A.M., CUISANCE D., REIFEN BERG J.M., FREZIL J.L., 1996. Emission de Trypanosoma congolense dans la salive et dans la goutte anale chez G lossina morsitans morsitans et G lossina tachinoides (Diptera : Glossinidae) au laboratoire. Revue Elev. Méd. vét. Pays trop., 42 : 132140.

14. GIBSON W.C., DUKES P., GASHU MBA J.K., 1988. Species-specific DNA probes for the identification of African trypanosomes in tsetse flies. Parasitology, 97: 63-73.

15. GOUTEUX J.P., GIBSON W.C., 1996. Detection of infections of Trypanosoma grayi in Glossina fuscipes fuscipes in the Central African Republic. Ann. trop. Med. Parasitol., 90: 555-557.

16. GRUVEL J., 1964. Contribution à l'étude écologique de Glossina tachinoides Westwood 1850 (Diptera, Muscidae) dans la réserve de Kalamalou, vallée du Bas-Chari. Thèse Doct., Université Paris VI, O rsay, France, $302 p$.

17. ITARD J., 1981. Les trypanosomoses animales africaines. In : Précis de parasitologie vétérinaire tropicale. Paris, France, Ministère de la Coopération et du Développement, p. 303-370. (Manuels et Précis d'élevage $n^{\circ} 10$ )

18. JEFFERIES D., HELFRICH M.P., MOLYNEUX D.H., 1987. Cibarial infections of Trypanosoma vivax and T. congolense in Glossina. Parasitol. Res., 7: 289-292.

19. KANMOGNE G.D., ASO NGANYI T., GIBSO N W.C., 1996. Detection of Trypanosoma brucei gambiense in serologically positive but aparasitaemic sleeping-sickness suspects in Cameroon, by PCR. Ann. trop. Med. Parasitol., 90: 475-483.

20. KIM MEL B.E., OLE-M OI-YOI O.K., YOUNG J.R., 1987. Ingi, a 5.2$\mathrm{kb}$ dispersed sequence element from Trypanosoma brucei that carries half of a smaller mobile element at either end and has homology with mammalian lines. Mol. Cell. Biol., 7: 1465-1475.

21. KRATZER R.D., ONDIEK F.O., 1989. The buffy-coat centrifugation technique, an improved method for the diagnosis of African trypanosomiasis. In : 20th Meeting CSIRTC, Monbasa, Kenya, April 10-14, 1989

22. KUKLA B.A., MAJIWA P.A.O., YOUNG J.R., MOLOO S.K., OLEMOI-YOI O.K., 1987. Use of species-specific DNA probes for the identification of trypanosome infection in tsetse flies. Parasitology, 95: $1-16$.

23. KUPPER W., STAAK C., SPATH J., 1990. Natural hosts of Glossina tachinoides (Diptera: Glossinidae) in N orthern Côte d'Ivoire. Trop. Med. Parasitol., 41: 217-218.

24. LAVEISSIERE C., BOREHAM P.F.L., 1976. Ecologie de Glossina tachinoides Westwood, 1850, en savane humide d'Afrique de l'O uest. I. Préférences trophiques. Cah. ORSTOM, Sér. Ent. méd. Parasitol., 16 : 187-200.

25. LAVEISSIERE C., GREBAUT $P_{\text {., }}$ 1990. Recherches sur les pièges à glossines (Diptera : Glossinidae). Mise au point d'un modèle économique : le piège «Vavoua ». Trop. Med. Parasitol., 41 : 185-192.

26. MCNAMARA J.J., LAVEISSIERE C., MASIGA D.K., 1995. Multiple trypanosome infections in wild tsetse in Côte d'Ivoire detected by PCR analysis and DNA probes. Acta trop., 59: 85-92.

27. MCNAMARA J.J., SNOW W.F., 1991. Improved identification of Nannomonas infections in tsetse flies from The Gambia. Acta trop., 48: 127-136.

28. MAJIWA P.A.O., MAINA M., WAITUMBI J.N., MIHOK S., ZWEYGART E., 1993. Trypanosoma (Nannomonas) congolense: molecular characterization of a new genotype from Tsavo, Kenya. Parasitology, 106: 151-162. 
29. MAJIW A P.A.O ., OLE-M OI-YOI O.K., NANTULYA V.M., 1993. N ew techniques for diagnosis of the African trypanosomiases. AgBiotech, News Inf., 5: 115-120.

30. MAJIWA P.A.O., OTIENO L.H., 1990. Recombinant DNA probes reveal simultaneous infection of tsetse flies with different trypanosomes. Mol. Biochem. Parasitol., 40: 245-254.

31. MASIGA D.K., MCNAMARA J.J., GIBSON W.C., 1996. A repetitive DNA sequence specific for Trypanosoma (Nannomonas) godfreyi. Vet. Parasitol., 62: 27-33.

32. MASIGA D.K., MCNAMARA J.J., LAVEISSIERE C., TRUC P., GIBSO N W.C., 1996. A high prevalence of mixed trypanosome infections in tsetse flies in Sinfra, Côte d'Ivoire, detected by DNA amplification. Parasitology, 112: 75-80.

33. MASIGA D.K., SMYTH A.J., HAYES P., BROMIDGE T.J., GIBSON W.C., 1992. Sensitive detection of trypanosomes in tsetse flies by DNA amplification. Int. J. Parasitol., 22: 909-918.

34. MATTIOLI R.C., JEAN O., BELEM A.M.G., 1990. Incidence de la trypanosomose sur la faune sauvage d'un ranch de gibier au Burkina Faso. Revue Elev. Méd. vét. Pays trop., 43 : 459-465.

35. MINTER-GO EBDLO ED E., LEAKE C.J., MINTER D.M., M CNAMARA J., KIMBER C., BASTIEN P., EVANS D.A., LE RAY D., 1993. Trypanosoma varani and T. grayi-like trypanosomes: development in vitro and in insect hosts. Parasitol. Res., 79: 329-333.

36. MOLOO S.K., GRAY M.A., 1989. N ew observations on the cyclical development of Trypanosoma vivax in Glossina. Acta trop., 4: 167-172.

37. NYEKO J.H.P., OLE-MOI-YOI O.K., MAJIWA P.A.O., OTIENO L.H., O CIBA P.M., 1990. Characterisation of trypanosome isolates from cattle in U ganda using species-specific DNA probes reveals predominance of mixed infections. Insect Sci. Appl., 11: 271-280.

38. PEN CHEN IER L., DUMAS V., GREBAUT P., REIFEN BERG J.M., CUNY G., 1996. Improvement of blood and fly gut processing for PCR diagnosis of trypanosomosis. Parasite, 4: 387-389.

39. REIFENBERG J.M., 1996. Etude des relations parasites-hôtes dans l'épidémiologie moléculaire des trypanosomoses bovines au Burkina Faso. Thèse doct., U niversité M ontpellier II, Montpellier, France, 151 p.

40. REIFEN BERG J.M., CUISANCE D., FREZIL J.L., CUNY G., DUVALLET G. Comparison of the susceptibility of different Glossina species to simple and mixed infections with Trypanosoma (Nannomonas) congolense Savannah and Riverine-Forest types. Med. vet. Entomol. (sous presse)
41. REIFEN BERG J.M., CUISANCE D., GIDUDU A., CUNY G., DUVALLET G., FREZIL J.L., 1996. Evaluation de la capacité vectorielle de Glossina tachinoides (Diptera, Glossinidae) vis-à-vis de Trypanosoma (Nannomonas) congolense : implications épidémiologiques. Parasite, $3: 267-276$.

42. REIFENBERG J.M., SOLANO P., DUVALLET G., CUISANCE D., SIMPORE J., CUNY G. Molecular characterization of the different $\mathrm{N}$ annomonas type trypanosomes isolated around Bobo-Dioulasso, Burkina Faso, Vet. Parasitol. (sous presse)

43. ROBERTS C.J., GRAY A.R., 1972. A comparison of Glossina morsitans submorsitans N ewst. and $G$. tachinoides W est., collected and maintained under similar conditions, as vectors of Trypanosoma (Nannomonas) congolense, T. (N.) simiae and T. (Duttonella) vivax. Ann. trop. Med. Parasitol., 66: 41-53.

44. SAIKI R.K., GELFAND D.H., STOFFEL S., SCHARF S.J., HIGUCHI R., HORN G.T., MULLIS K.B., ERLICH H.A., 1987. Primer-directed enzymatic amplification of DNA with a thermostable DNA polymerase. Science, 239: 487-491

45. SCHARES G., MELHITZ D., 1996. Sleeping sickness in Zaire: a nested polymerase chain reaction improves the identification of Trypanosoma (Trypanozoon) brucei gambiense by specific kinetoplast DNA probes. Trop. Med. int. Health, 1: 59-70.

46. SOLANO P., ARGIRO L., REIFENBERG J.M., YAO Y., DUVALLET G., 1995. Field application of the polymerase chain reaction $(P C R)$ to the detection and characterization of trypanosomes in Glossina longipalpis (Diptera: Glossinidae) in Côte d'Ivoire. Mol. Ecol., 4: 781-785.

47. SO LAN O P., REIFEN BERG I.M., AM SLER-DELAFO SSE S., KABO RE I., CUISAN CE D., DUVALLET G., 1996. Trypanosome characterization by polymerase chain reaction in Glossina palpalis gambiensis and Glossina tachinoides from Burkina Faso. Med. vet. Entomol., 10: 354-358.

48. VERY P., BOCQUENTIN R., DUVALLET G., 1990. Sensibilité de la double centrifugation pour la recherche des trypanosomes. Revue Elev. Méd. vét. Pays trop., 43 : 325-329.

49. WoO P.T.K., 1970. The haematocrit centrifuge technique for the diagnosis of African trypanosomiasis. Acta trop., 27: 384-386.

50. WO O LHOUSE M.E.J., MCNAMARA J.J., HARGROVEJ.W., BEALBY K., 1996. Distribution and abundance of trypanosome (subgenus Nannomonas) infections of the tsetse fly Glossina pallidipes in Southern Africa. Mol. Ecol., 5: 11-18.

Reçu le 3.4.97, accepté le 26.6.97 


\section{Summary}

Reifenberg J.M., Solano P., Bauer B., Kabore I., Cuny G., D uvallet G., Cuisance D. Advantage of the PCR technique in assessing the epidemiology of bovine trypanosomosis: example of the agropastoral development area of Yalé, Burkina Faso

The PCR (polymerase chain reaction) technique was used to identify trypanosomes both in tsetse and infected cattle in the agropastoral development area of Yalé in southern Burkina Faso. $O$ ut of 84 parasitologically positive midguts of Glossina tachinoides, 50 (Trypanosoma congolense savannah and riverine forest types, T. simiae and T. vivax) were identified by PCR. U sing PCR on bovine blood samples, it was found that $T$. congolense savannah type and $T$. vivax were predominant, while the riverine forest type of $T$. congolense could not be detected. Some aparasitaemic but clinically suspected animals reacted positively when specific primers for the savannah type of $T$. congolense were used. The results confirmed the high potential of the PCR technique in detecting cryptic and/or mixed infections in the different hosts. Added to previous studies describing comparable approaches, the present results show that the tools using molecular biology can provide valuable information on the complex relationships between the savannah or riverine forest types of T. congolense and their vectors, and also their vertebrate hosts. This probably enables the molecular markers to be considered as pathogenicity markers. Their diagnostic capability and their helpful part in approaching the parasite/ host relationship for better control in the field are discussed.

Key words: Cattle - Glossina - Trypanosomosis - Trypanosoma congolense - Trypanosoma simiae - Trypanosoma vivax - Polymerase chain reaction - Molecular probe - Host pathogen relation - Vector - Epidemiology - Burkina Faso.

\section{Resumen}

Reifenberg J.M., Solano P., Bauer B., Kabore I., Cuny G., Duvallet G., Cuisance D. Aporte de la técnica PCR para una mejor comprensión de la epizootiología de las tripanosomiasis bovinas: ejemplo de la zona de cultivo pastoril de Yalé en Burkina Faso

Se utilizó la técnica de $P C R$ (reacción en cadena de polimerasas) para la identificación de tripanosomas en glosinas y bovinos infectados, originarios de la zona de cultivo pastoril de Yalé, al sur de Burkina Faso. De los 84 intestinos medios parasitológicamente positivos para G lossina tachinoides que se analizaron, 50 fueron identificados mediante PCR para (Trypanosoma congolense tipos « savana » y « bosque », T. simiae y T. vivax). En los bovinos la técnica PCR demostró la predominancia de T. congolense « savana »y de T. vivax. El taxon «bosque » de T. congolense no se detectó en el ganado. Ciertos animales aparasitémicos pero sospechosos mostraron señales positivas mediante el PCR con los cebos específicos para T. congolense " savana ». Estos resultados confirman el gran interés de la técnica PCR para revelar las « pauci infecciones » y las infecciones mixtas en los diferentes huéspedes. Al igual que aplicaciones similares publicadas anteriormente, este estudio demuestra el aporte de los instrumentos de la biología molecular para la demostración de relaciones complejas de afinidad de los taxones « savana » $y$ « bosque » de $\mathrm{T}$. congolense con respecto a sus vectores, pero también con respecto a los huéspedes vertebrados, confiriendo, aparentemente, a los marcadores moleculares el valor de marcadores de poder patógeno. Se discuten los puntos relativos al valor diagnóstico y a la contribución para comprender mejor las relaciones huésped-parásito con vistas a una lucha de campo más eficaz.

Palabras clave: Ganado bovino - Glossina - Tripanosomosis Trypanosoma congolense - Trypanosoma simiae Trypanosoma vivax - Reacción de cadenas de polimerasa Sonda molecular - Relación huesped patógeno - Vector Epidemiología - Burkina Faso. 